\title{
Pharmacokinetic and Pharmacodynamic Interactions with Levofloxacin in Renal Transplant Patients with Suspected Pneumonia
}

\author{
A. Scott Mathis* and Daryl S. Schiller
}

Department of Pharmacy, Saint Barnabas Medical Center, Livingston, New Jersey, USA

\begin{abstract}
Ciprofloxacin, a fluoroquinolone antibiotic, has been linked to an increased risk of acute cellular rejection and drug interactions with the calcineurin inhibitors in renal transplant recipients. Little is known about the effects of levofloxacin. We report our evaluation for pharmacokinetic and pharmacodynamic interaction between levofloxacin and the calcineurin inhibitors. A retrospective review was conducted of renal transplant recipients with known or suspected community acquired pneumonia who received levofloxacin. Patients were assessed for rejection at 90-day follow-up, and compared to a historical ciprofloxacin cohort. Assessment of the drug-drug interaction between levofloxacin and the calcineurin inhibitors required stable dose of the immunosuppressants, a baseline trough level, and follow-up levels. Rejection occurred in 1 of the 26 included patients (3.8\%), less than the rate in the historical ciprofloxacin group at 4 weeks $(28.6 \% ; \mathrm{p}=0.012)$ and 12 weeks $(45.2 \% ; \mathrm{p}<0.001)$. There was no significant drug-drug interaction between levofloxacin and cyclosporine $(n=8)$ or tacrolimus $(n=6)$ when trough levels were evaluated. In summary, renal transplant patients receiving levofloxacin for pneumonia did not appear to have a temporally-related elevated risk of ACR or a pharmacokinetic drug-drug interaction with the calcineurin inhibitors.
\end{abstract}

\section{INTRODUCTION}

Levofloxacin is a commonly used fluoroquinolone antibiotic recommended for the empiric management of community acquired pneumonia (CAP) in inpatients and outpatients [1]. In renal transplant recipients, levofloxacin is utilized due to broad coverage against common organisms and the perception that it is safer compared with other agents with more nephrotoxicity (aminoglycosides) or drug interactions (macrolides) [2]. However, quinolone antibiotics appear to possess immunomodulatory properties [3,4], and ciprofloxacin exposure appeared to increase the risk of acute cellular rejection in at least one study [5]. Furthermore, little is known about the pharmacokinetic drug-drug interaction between levofloxacin and the calcineurin inhibitors.

We sought to evaluate the relationship between levofloxacin and the risk of developing acute cellular rejection (ACR) or a pharmacokinetic drug-drug interaction with the calcineurin inhibitors (CNIs) after an episode of suspected pneumonia in renal transplant or simultaneous pancreaskidney recipients $[5,6]$.

\section{METHODS}

A retrospective medical record review was conducted after approval from the Institutional Review Board. Relevant hospital admissions were identified through a computerized database search of charges for levofloxacin and diagnosis codes for prior renal or kidney/pancreas transplant during the calendar years 1999 and 2000. The dates were chosen because the transplant program used both calcineurin inhibitors

*Address correspondence to this author at the Department of Pharmacy, Saint Barnabas Medical Center, Livingston, New Jersey, USA;

E-mails: smathis@sbhcs.com and amathis100@aol.com in nearly equal distribution at the time. All of the following inclusion criteria were needed for the assessment of ACR: adult ( $\geq 18$ years) renal or kidney/pancreas transplant recipients who were hospitalized and received levofloxacin for suspected CAP and had a 90-day outpatient follow-up visit. Patients were not excluded as long as inclusion criteria were met. Included patients were evaluated for the duration of the CAP-related admission.

After discharge, the presence or absence of ACR was recorded within 90 days of follow-up utilizing outpatient and inpatient records. Only ACR episodes occurring during the 90 day post-levofloxacin exposure were considered. ACR rates were compared to a published historical control group who received ciprofloxacin, and had a similar follow-up [5]. ACR was considered present if biopsy-proven and assessed according to the Banff criteria [7]. Daily cyclosporine or tacrolimus concentrations were evaluated to determine the presence or absence of an interaction with levofloxacin. $\mathrm{Pa}$ tients were included in the drug-drug interaction assessment if an appropriately-timed (i.e., 11-12 hours after the last dose, at steady state) baseline calcineurin inhibitor trough level was available on the day of levofloxacin initiation, but prior to the start of the agent. Additional considerations were a stable calcineurin inhibitor dose from day-to-day and available follow-up levels. Cyclosporine levels were tested using a TDx analyzer, and tacrolimus using an IMx analyzer (both Abbott Laboratories). Demographic data were collected to determine patient baseline characteristics in the group evaluated for ACR and the patients studied for a drugdrug interaction with cyclosporine or tacrolimus. A validated prediction rule, the Fine score, was used to provide baseline risk assessment for patients suspected to have CAP [7-9]. Pneumonia outcomes were also evaluated. 


\section{STATISTICS}

Based on the prior work with ciprofloxacin, it was determined that 24 patients would be needed, due to an expected reduction of acute cellular rejection from 29\% (ciprofloxacin rate) to $2 \%$ (expected rate in the population) at 4 weeks with $80 \%$ power, and an $\alpha$ of 0.05 . The proportions were compared using Chi-squared or Fisher's exact test, continuous demographic characteristics were compared using the Mann-Whitney U test, and the ANOVA test was utilized to evaluate drug levels versus baseline. Pearson correlation was used to relate risk score to length of stay and requirement for other antibiotics. Statistical analysis was performed on SPSS statistical package version 12 (SPSS Inc., Chicago IL). A $p<0.05$ was considered statistically significant.

\section{RESULTS}

A total of 377 admissions were identified in which transplant patients received levofloxacin during the studied years. Of those admissions 46 involved suspected CAP and 26 had the required 90-day follow-up in the outpatient clinic. Among patients excluded for insufficient available followup, $18(90 \%)$ were lost to follow-up and $2(10 \%)$ patients expired. Patients who died did not have an ACR episode. The culture results among those excluded were as follows: 4 (20\%) were diagnosed with Aspergillus species, 1 (5\%) with Candida species, 1 (5\%) with Pneumocystis carinii, 3 (15\%) with Staphylococcus species, and 1 (5\%) with Streptococcus pneumoniae.

Of the 26 patients included, only 9 (34.6\%) had an organism isolated, and 5 of those were opportunistic infections (19.2\% overall, $55.6 \%$ of positive cultures). The opportunistic infections included Aspergillus species with Mycoplasma pneumoniae $(\mathrm{n}=1)$, Aspergillus species alone $(\mathrm{n}=2)$ and Pneumocystis carinii $(\mathrm{n}=2)$. Chylamidia pneumoniae $(\mathrm{n}=1)$, Enterococcus faecalis $(\mathrm{n}=1)$, and Staphylococcus aureus $(n=2)$ were also identified. Fungal cultures usually took several weeks to return, so levofloxacin was not used concurrently with antifungal agents. Baseline and treatment characteristics are presented in Table 1. Patients were about 2 years post-transplant and mostly middle-aged male Caucasians. The majority of patients had a Fine score of II (46.2\%). Levofloxacin was initiated at a mean dose of $442 \mathrm{mg}(20$ patients received $500 \mathrm{mg}$ and 6 received $250 \mathrm{mg}$ - appropriate to their level of renal function), and continued for approximately 4 days. Other antibiotics were occasionally used to enhance the spectrum of activity. The average length of stay approached 10 days. All patients improved, but many were switched to antimicrobial agents other than levofloxacin. The Fine score did not correlate to length of hospital stay $(\mathrm{P}=0.56)$ or number of antibiotics needed $(\mathrm{P}=0.76)$.

During 90-day follow-up, 1 patient $(3.8 \%)$ developed ACR grade IIA 10 days after beginning levofloxacin. No other patients had known or suspected ACR. Compared with a historical ciprofloxacin group, the 4-week (28.6\%, $\mathrm{p}=0.012)$ and 12-week $(45.2 \%, \mathrm{p}<0.001)$ incidence of ACR was less frequent (Fig. 1) [5].

The assessment of a drug-drug interaction between levofloxacin and the calcineurin inhibitors demonstrated a slight, but non-significant increase between baseline and follow-up cyclosporine (Fig. 2) or tacrolimus (Fig. 3) trough levels.

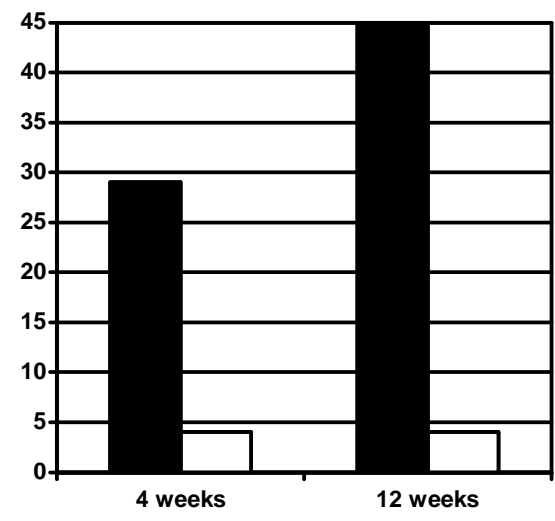

Ciprofloxacin QLevofloxacin

Fig. (1). Percent of Patients with ACR at 4 weeks and 12 weeks after Levofloxacin (present study) or Ciprofloxacin (historical). Ciprofloxacin ACR rates (black bars) were $28.6 \%$ at 4 weeks and $45.2 \%$ at 12 weeks. Levofloxacin ACR rates (white bars) were $3.8 \%$ at 4 weeks $(\mathrm{p}=0.012)$ and $3.8 \%$ at 12 weeks $(\mathrm{p}<0.001)$.

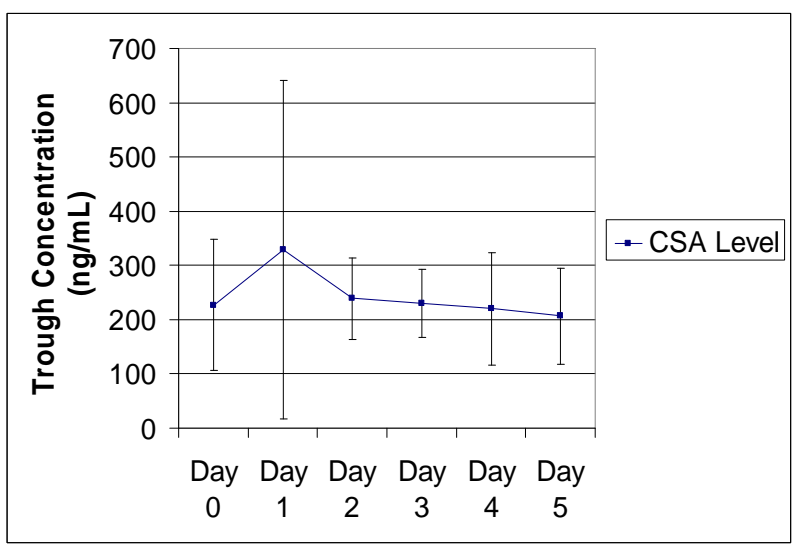

Fig. (2). Cyclosporine (CSA) Trough Levels Before (Day 0) and During (Days 1-5) Levofloxacin Exposure. ANOVA p= NS.

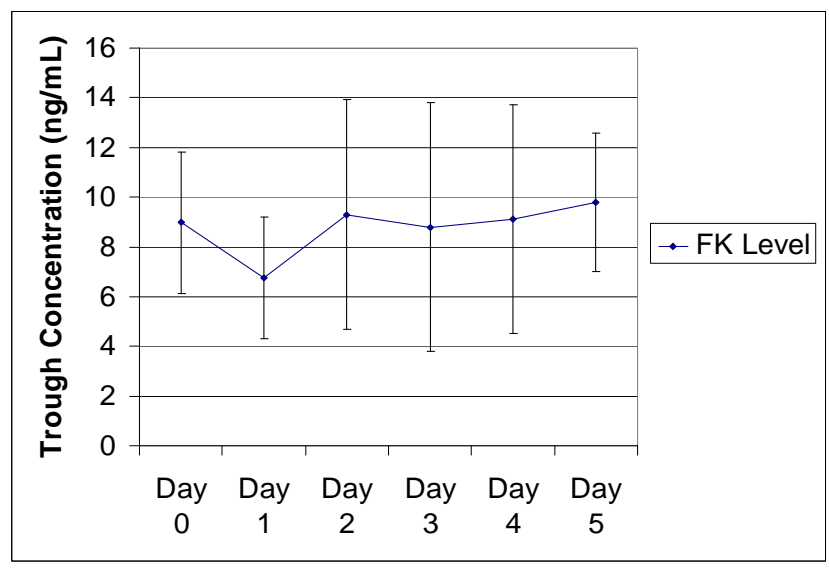

Fig. (3). Tacrolimus (FK) Trough Levels Before (Day 0) and During (Days 1-5) Levofloxacin Exposure. ANOVA p= NS.

\section{DISCUSSION}

The main findings of this retrospective study were an apparent lack of influence of levofloxacin on the incidence 
Table 1. Demographics and Treatment Characteristics of Patients Evaluated for Acute Cellular Rejection or LevofloxacinCalcineurin Inhibitor Drug Interaction

\begin{tabular}{|c|c|c|c|c|}
\hline & $\begin{array}{l}\text { Patients Evaluated } \\
\text { for ACR }(n=26)\end{array}$ & $\begin{array}{c}\text { Cyclosporine } \\
\text { Interaction }(n=8)\end{array}$ & $\begin{array}{c}\text { Tacrolimus } \\
\text { Interaction }(n=6)\end{array}$ & $\begin{array}{c}\text { P-value Cyclosporine vs. } \\
\text { Tacrolimus }\end{array}$ \\
\hline Age, mean (SD) & $51.4(14.8)$ & $60(14)$ & $48(10)$ & 0.108 \\
\hline Sex (male), n (\%) & $20(76.9)$ & $7(87.5)$ & $5(83.3)$ & 0.999 \\
\hline Ethnicity, n (\%) & & & & 0.177 \\
\hline Caucasian & $16(61.5)$ & $7(87.5)$ & $2(33.33)$ & \\
\hline African American & $7(26.9)$ & $1(12.5)$ & $2(33.33)$ & \\
\hline Other & $3(11.6)$ & $0(0)$ & $2(33.33)$ & \\
\hline Transplant type, n (\%) & & & & 0.287 \\
\hline Cadaveric & $16(61.5)$ & $5(62.5)$ & $2(33.3)$ & \\
\hline Living donor & $9(34.7)$ & $3(37.5)$ & $3(50)$ & \\
\hline Simultaneous pancreas/kidney & $1(3.8)$ & $0(0)$ & $1(16.7)$ & \\
\hline Days post-transplant, mean (SD) & $837.1(1210)$ & $1565.6(1842.4)$ & $463.7(389.1)$ & 0.282 \\
\hline Immunosuppression, $\mathrm{n}(\%)$ & & & & Not Applicable \\
\hline Cyclosporine/MMF/prednisone & $13(50)$ & $7(87.5)$ & $0(0)$ & \\
\hline Cyclosporine/AZA/prednisone & $1(3.8)$ & $1(12.5)$ & $0(0)$ & \\
\hline Cyclosporine/SIR/prednisone & $1(3.8)$ & $0(0)$ & $0(0)$ & \\
\hline Tacrolimus/MMF/prednisone & $11(42.4)$ & $0(0)$ & $6(100)$ & \\
\hline Fine Risk Score, mean (SD) & $73.64(48)$ & $89(42.1)$ & $67(45.7)$ & 0.282 \\
\hline Fine Risk Group, n (\%) & & & & 0.552 \\
\hline II & $12(46.2)$ & $2(25)$ & $3(50)$ & \\
\hline III & $2(7.7)$ & $1(12.5)$ & $0(0)$ & \\
\hline IV & $8(30.8)$ & $4(50)$ & $3(50)$ & \\
\hline $\mathrm{V}$ & $3(11.5)$ & $1(12.5)$ & $0(0)$ & \\
\hline Unable to calculate & $1(3.8)$ & $0(0)$ & $0(0)$ & \\
\hline Levofloxacin dose, mean (SD) & $442.3(107.4)$ & $468.8(88.4)$ & $500(0)$ & 0.755 \\
\hline Days of levofloxacin, mean (SD) & $4.27(2.3)$ & $3.13(1.8)$ & $4.33(2.3)$ & 0.345 \\
\hline Additional antibiotics, mean (SD) & $0.43(1.1)$ & $0.13(0.4)$ & $0.17(0.4)$ & 0.95 \\
\hline Length of stay, mean (SD) & $9.65(10.6)$ & $4.88(1.9)$ & $6.33(4)$ & 0.662 \\
\hline
\end{tabular}

$\mathrm{AZA}=$ azathioprine; $\mathrm{MMF}=$ mycophenolate mofetil; $\mathrm{SIR}=$ sirolimus; $\mathrm{SD}=$ standard deviation .

of ACR or the trough levels of cyclosporine and tacrolimus. We acknowledge the limitations associated with this type of retrospective study. However, we used objective markers such as CNI levels and biopsy-proven rejection episodes, and carefully evaluated both inpatient and outpatient records during the follow-up period. Additionally, we only considered events occurring during the follow-up period.

The historical comparator group used for the study indicated an increased risk of ACR after ciprofloxacin exposure in renal transplant recipients, with higher-than-expected
ACR rates during available follow-up [5]. The incidence of ACR in our population met the expected rate in the population, suggesting that levofloxacin did not increase the risk of ACR. Some important differences should be considered when comparing the 2 studies, and may serve as limitations when interpreting the data. First, the ciprofloxacin patients were within the first 6 months post-transplant and indications other than pneumonia were treated. We studied only patients with pneumonia because of the high risk for an adverse outcome. Thus, due to the infrequent occurrence of pneumonia 
in the renal transplant population, the mean time posttransplant was 2.3 years among our patients [10]. Second, most of our patients received mycophenolate mofetil (MMF) as the antiproliferative agent, whereas azathioprine was used in the ciprofloxacin study. It is possible that mycophenolate mofetil more effectively prevented rejection than azathioprine [11]. Still, our study provides reassurance that levofloxacin can be used without fear of an increased risk of ACR under a CNI-MMF regimen. Demographic characteristics provided in the ciprofloxacin publication were limited, but appeared to be similar to the present study, suggesting that the patients may have been similar, even though the post-transplant time-frame was different [5].

The reported mechanism for ciprofloxacin-related ACR is an increase in interleukin-2 (IL-2) release [4]. This may be related to a number of factors including the ability of ciprofloxacin to upregulate nuclear factor of activated T-cells (NFAT) [3]. This would allow ciprofloxacin to bypass the calcineurin inhibition pathway for cyclosporine and still stimulate IL-2 production [12]. Increased IL-2 production has been associated with levofloxacin in in vitro assays, however, data indicate that quinolone agents with a cyclopropyl moiety (ciprofloxacin, moxifloxacin) lead to a more pronounced immunomodulatory/ immunostimulant effect than agents without a cyclopropyl moiety (levofloxacin, norfloxacin) [3].

Some fluoroquinolone antibiotics inhibit cytochrome P450-mediated drug metabolism and increase the risk of a drug-drug interaction with cyclosporine [13]. A single-dose pharmacokinetic study in healthy volunteers did not demonstrate a pharmacokinetic drug-drug interaction between levofloxacin and cyclosporine [6]. Another recent pharmacokinetic study performed in renal transplant patients documented a levofloxacin-induced increase in cyclosporine AUC $(25 \%)$ and trough levels, and tacrolimus AUC (27\%) [14]. For unclear reasons, the authors of the transplant study used levofloxacin 500mg twice daily for 6 days for urinary tract infection, and performed 11 blood samples over 12 hours on the $6^{\text {th }}$ day [14]. The results of the renal transplant pharmacokinetic study suggest the possibility of an increase in CNI due levofloxacin [14] which cannot be excluded by our data. However, our results demonstrate a general lack of interaction between cyclosporine or tacrolimus with levofloxacin when doses commonly used in clinical practice are given. Both transplant studies had a similar sample size so it is possible that higher doses have a greater effect [14]. It is also possible, but less likely, that the longer duration of levofloxacin administration in the prospective study (6 vs. 5 days) accounted for the difference. Still, our study was limited by a small sample size. A slight first-dose effect was observed with cyclosporine levels, and an upward trend with tacrolimus levels, suggesting that we cannot exclude the possibility that some patients may experience an interaction. In fact, the wide variance in data on day 1 with cyclosporine suggests a differential response, where some patients may experience a change in level, and others do not. Although our study is limited by the retrospective nature, we were careful to include only appropriately-timed levels drawn during stable calcineurin inhibitor dosing. There appears to be little likelihood that levofloxacin results in any direct cytochrome P450 interactions, but there are other possible ex- planations [6]. P-glycoprotein and possibly other transporters have an important role in the disposition of levofloxacin, cyclosporine and tacrolimus and could result in a change in drug levels $[15,16]$. Alternatively, the infection itself could stimulate release of cytokines, such as IL-6, that may inhibit cytochrome P450 enzymes [17]. It is also possible that quinolone-mediated cytokine release could have the same effect [3].

\section{CONCLUSION}

Renal transplant patients receiving levofloxacin for pneumonia did not appear to have a temporally-related elevated risk of ACR or a pharmacokinetic drug-drug interaction with the calcineurin inhibitors when used at approved doses. These results do not exclude the possibility of a pharmacokinetic interaction in some patients.

\section{ACKNOWLEDGEMENT}

The authors wish to thank Staci Siconolfi, Pharm.D. for assistance with data collection.

\section{REFERENCES}

[1] Mandell LA, Wunderink RG, Anzueto A, et al. Infectious diseases society of America/American thoracic society consensus guidelines on the management of community-acquired pneumonia in adults. Clin Infect Dis 2007; 44 (Suppl 2): S27-72.

[2] Fishman JA, Rubin RH. Infection in organ-transplant recipients. N Engl J Med 1998; 338: 1741-51.

[3] Dalhoff A, Shalit I. Immunomodulatory effects of quinolones. Lancet Infect Dis 2003; 3: 359-71.

[4] Riesbeck K, Gullberg M, Forsgren A. Evidence that the antibiotic ciprofloxacin counteracts cyclosporine-dependent suppression of cytokine production. Transplantation 1994; 57: 267-72.

[5] Wrishko RE, Levine M, Primmett DRN, et al. Investigation of a possible interaction between ciprofloxacin and cyclosporine in renal transplant patients. Transplantation 1997; 64: 996-9.

[6] Doose DR, Walker SA, Chien SC, et al. Levofloxacin does not alter cyclosporine disposition. J Clin Pharmacol 1998; 38: 90-3.

[7] Racusen LC, Solez K, Colvin RB, et al. The Banff 97 working classification of renal allograft pathology. Kidney Int 1999; 55: 713-23.

[8] Fine MJ, Smith MA, Carson CA, et al. Prognosis and outcomes of patients with community-acquired pneumonia. JAMA 1996; 275 : 134-41.

[9] Fine MJ, Auble TE, Yealy DM, et al. A prediction rule to identify low-risk patients with community-acquired pneumonia. N Engl J Med 1997; 336: 243-50.

[10] Tveit DJ, Hypolite IO, Poropatich RK, et al. Hospitalizations for bacterial pneumonia after renal transplantation in the United States. J Nephrol 2002; 15: 255-62.

[11] Halloran P, Mathew $\mathrm{T}$, Tomlanovich S, et al. Mycophenolate mofetil in renal allograft recipients: a pooled efficacy analysis of three randomized, double-blind, clinical studies in prevention of rejection. The International Mycophenolate Mofetil Renal Transplant Study Groups. Transplantation 1997; 63: 39-47.

[12] Rao A. NFATp, a cyclosporin-sensitive transcription factor implicated in cytokine gene induction. J Leukoc Biol 1995; 57: 536-42.

[13] McLellan RA, Drobitch RK, Monshouwer M, et al. Fluoroquinolone antibiotics inhibit cytochrome P450-mediated microsomal drug metabolism in rat and human. Drug Metab Dispos 1996; 24: 1134-8.

[14] Federico S, Carrano R, Capone D, et al. Pharmacokinetic interaction between levofloxacin and ciclosporin or tacrolimus in kidney transplant recipients. Ciclosporin, tacrolimus and levofloxacin in renal transplantation. Clin Pharmacokinet 2006; 45: 169-75.

[15] Sikri V, Pal D, Jain R, et al. Cotransport of macrolide and fluoroquinolones, a beneficial interaction reversing P-glycoprotein efflux. Am J Ther 2004; 11: 433-42. 
[16] Yamaguchi H, Yano I, Saito H, et al. Pharmacokinetic role of Pglycoprotein in oral bioavailability and intestinal secretion of grepafloxacin in vivo. J Pharmacol Exp Ther 2002; 300: 1063-9.
[17]

Liao JS, Reiss WG. Drug-cytokine interactions: focus on cyclosporine. Pharmacotherapy 1996; 16: 401-8.

Received: April 4, 2008

(c) Mathis and Schiller; Licensee Bentham Open.

This is an open access article licensed under the terms of the Creative Commons Attribution Non-Commercial License (http://creativecommons.org/licenses/ by-nc/3.0/) which permits unrestricted, non-commercial use, distribution and reproduction in any medium, provided the work is properly cited. 